\title{
SELECTIVE IGM DEFICIENCY: FOLLOW-UP AND OUTCOME
}

\author{
Canan Caka ${ }^{1}$, özlem satırer ${ }^{1}$, pınar kahyaoglu ${ }^{1}$, İhan Tezcan ${ }^{2}$, and Deniz Cagdas ${ }^{1}$ \\ ${ }^{1}$ Hacettepe University \\ ${ }^{2}$ Hacettepe Universitesi Tip Fakultesi
}

October 20, 2020

\begin{abstract}
Background: Selective IgM deficiency ( $\operatorname{sgMD}$ ), classified under primary immunodeficiencies, is characterized by low serum $\operatorname{IgM}(<2$ SD for age $)$, normal IgG and IgA levels. The aim of this study was to define immunologic and clinical features of sIgMD. Method: We assessed a retrospective medical record of patients who fullfilled the criteria for sIgMD in a Pediatric Immunology department. Results: There were 55 patients with sIgMD. Out of 55 patients, thirteen(23.6\%) diagnosed with a well-defined primary immunodeficiency (PID) during the follow-up.The ratio of the sIgMD was \%0.12 in the out-patient clinic of pediatric immunology. Out of 33 patients, $8(23.5 \%)$ were asymtomatic during the follow-up period. Fifteen(45.4\%) patients presented with several type of infections). Six patients (18\%) had chromosomal anomaly, or syndrome (trisomy 21 , 22q11.2 deletion 1p deletion, CHARGE syndrome, and Cohen Syndrome). Six (18\%) had autoimmune/inflammatory diseases, such as Behcet's disease, immune thrombocytopenic purpura, Crohn disease, Guillain Barre syndrome, and diabetes mellitus. Five(15\%) had allergic disorders. Three(9\%) have developed malignancy. The diagnoses of thirteen PID patients were combined immunodeficiency, common variable immunodeficiency, autoimmune lymphoproliferative syndrome, chronic granulomatous disease, adenosine deaminase deficiency, and congenital neutropenia. Genetic disorders, autoimmune/inflammatory and allergic diseases may accompany sIgMD. Approximately one third of the patients were asymptomatic in our series. Malignancy risk is relatively increased. We observed that an important ratio of patients with low IgM (23.6\%) got sooner the diagnosis of a specific PID in the follow-up period. Conclusion: Thus, patients with sIgMD should be followed regularly in immunology clinics.
\end{abstract}

\section{Introduction}

Selective IgM deficiency (sIgMD) is a rare form of dysgammaglobulinemia, It is classified under primary humoral immunodeficiencies and characterized by low serum level of IgM, normal IgG and IgA levels with normal $\mathrm{T}$ cell numbers and function in the absence of a defined immunodeficiency. Pathogenesis of sIgMD is unknown, and no genetic or molecular basis has been defined yet. However, there are some hypothesis suggesting that it may be due to defective $\mathrm{B}$ cell maturation or $\mathrm{T}$ cell dysfunction $[1,2]$.

The European Society for Immunodeficiencies (ESID) Registry defines primary selective IgM deficiency (sIgMD) as serum IgM level repeatedly below 2 standard deviations $(<-2 \mathrm{SD})$ of reference levels, and normal levels of serum IgA, IgG and IgG subclasses, normal vaccination responses, absence of $\mathrm{T}$ cell defects and absence of causative external factors [3-5]. In another definition, sIgMD was defined as having low IgM, which is usually lower than $20 \mathrm{mg} / \mathrm{dL}$ in infants and children, or lower than 2SD. Usually, serum IgM levels are lower than10-20 mg/dL. The levels of other immunoglobulin isotypes are typically normal, although IgE may be increased [6, 7]. There is no definition for IgE, and high IgE may be observed in some cases [8]. SIgMD is observed in both children and adults [9]. 
Although it may occur in asymptomatic individuals [10], patients with sIgMD may present with various clinical features. It may associate with recurrent upper respiratory tract infections (URTI), atopic manifestations (rhinitis, asthma etc.), autoimmune diseases, malignancies, and other primary humoral immunodeficiency diseases. There are several reports in the medical literature including diseases that may cause decreased IgM levels, such as recurrent infections, autoimmune diseases (Celiac disease, hemolytic anemia), and different types of primary immunodeficiency (PID) (thymic hypoplasia, Wiskott Aldrich syndrome, ataxia telangiectasia, common variable immunodeficiency (CVID), Bruton agammaglobulinemia and congenital diseases (Bloom syndrome, Russel silver syndrome, DiGeorge syndrome) [11].

Our aim is evaluation of the demographic features, clinical presentations, associated diseases and infections of the patients who are followed up with sIgMD.

\section{Patients and Methods}

The study was conducted in Hacettepe Üniversity İhsan Dogramacı Children's Hospital, Ankara, Turkey. The hospital serves as a referral tertiary hospital. We assessed a retrospective medical record of 27,000 patients who admitted to Pediatric Immunology department. To define selective IgM deficient patients, The European Society for Immunodeficiencies (ESID) Registry criteria have been used [3-5]. Patients who had repeatedly (at least twice in a 6 -month period) low serum IgM level according to age referrals were evaluated in the study. Fifty-five patients were found in this cohort (Figure 1). Thirteen patients who were diagnosed with a primary immunodeficiency had also low IgM levels with normal IgG and IgA levels. Forty-two patients fulfilling the diagnosis of sIgM deficiency were evaluated over a five-year period (2013-2018).

As transient hypogamaglobulinemia may prolong to five years of age[12], we determined the patients who got the diagnosis less than five years of age. Eighteen of 42 patients had taken the diagnosis of sIgMD before five years of age. Out of 18, in nine patients sIgM deficiency continued after five years of age, in two patients IgM levels normalized, and seven patients lost follow-up. Eventually, 33 patients were fulfilling the disease criteria [13].

Cases of sIgMD were reviewed for the symptoms on admission, concurrent conditions, and clinical course. Patients with genetic mutations, chromosomal abnormalities and associated immunological disorders were also recorded. Laboratory findings; complete blood counts, serum immunoglobulin levels and lymphocyte subsets were noted. To evaluate vaccination response antiHbs antibody and isohemaglutinins were used.

The patients with PID $(n=13)$ and patients who got the diagnosis of sIgMD at less than 5 years of age $(n=9)$ were evaluated separately (Figure 1), and their characteristics are given after the results of patients with sIGMD.

The study was approved by the ethical committee of Hacettepe University Medical School Hospital (GO 18/182-20).

\section{Statistical analysis}

All the data collected during the study were uploaded to SPSS statistical program. Parametrical tests (Student t-test) were used to analyze the variables which distributed normally. The variables that did not have normal distribution were analyzed by nonparametric tests (Mann-Whitney U test). Categorical and qualitative variables were analyzed by Fisher Exact test and Chi-Square test. A $p$ value lower than 0.05 was considered to be statistically significant. 


\section{RESULTS}

Demographic findings: In our study, the ratio of SIgMD is found as $0.12 \%$ among the patients that were followed up in pediatric immunology out-patient clinic for a definite period (33 out of 27,000 patients) Out of 33 patients, 25 patients $(74 \%)$ were male and $8(26 \%)$ were female. The median age of onset of disease was 3 years (1-33), and the median age of diagnosis was 8 years (1-43). Six out of 33 patients (18.2\%) were adult, 27 of them $(81.8 \%)$ were children. Age at diagnosis and gender of each patients were given in supplementary table 2 .

\section{Clinical Findings:}

Clinical Presentation: Most common form of presentation was pneumonia (26,4\%), skin infection (Herpes simplex, Varicella zoster, Human papilloma virus infections) (17,6\%), URTI (11\%), lymphadenopathy $(5,8 \%)$, meningitis $(5,8 \%)$, and oral mucocutaneous candidiasis $(2.3 \%)$ and lichen planus $(2,9 \%)$. Recurrent respiratory tract infection was the most common complaint that the patients were referred to the immunology department before we exclude patients who were under five years old. Afterwards, frequency of presentation with recurrent URTI decreased significantly (11 (26\%) versus $4(12 \%))$. Clinical presentation findings of patients were given in Table 1 .

Four patients were asymptomatic, and diagnosed with sIgMD incidentally. Among all, two patients have history of immunodeficiency in their family. There were five patients who had family history of increased susceptibility to infections or immunodeficiencies. Five patients had syndromic facial features, and two of them were diagnosed with trisomy 21, one was diagnosed with DiGeorge syndrome, one diagnosed with Charge syndrome, and one with Cohen syndrome (Two more patients were diagnosed with DiGeorge syndrome among sIgMD patients who ara still under five years of age so were not included as sIgMD) Cardiac disease (Patent foramen ovale, mitral and aortic valve insufficiency, mitral valve prolapse, left ventricular hypertrophy, atrial septal defect, patent ductus arteriosus) was seen in seven (21.2\%) patients. One of the patients admitted to hospital at the age of 2 months with chickenpox. He is still being followed up for isolated IgM deficiency. Associating diseases of sIgMD patients at presentation and follow-up are shown in Table 1 and 2 respectively.

\section{Diseases in the Follow-up}

Infectious and noninfectious diseases of patients during follow-up period were given in supplementary table 2 .

Infections: During the follow-up, recurrent URTI (60.6\%) was the most common finding followed bypneumonia $(36,3 \%)$. The frequencies of infections in sIgMD are shown in Table 3.

Malignancy: Malignancy developed in three (9\%) out of 33 patients. These were acute myeloid leukemia $(\mathrm{n}=2)$, and tubular adenoma in sigmoid colon $(\mathrm{n}=1)$. A 4 year-old patient presented with neuroblastoma, and after four years of follow-up, AML developed in the same patient. He was treated successfully.

Autoimmune and Inflammatory Diseases: Six patients with sIgMD was additionally diagnosed with autoimmune and inflammatory diseases, such as, Behcet's disease $(\mathrm{n}=2)$, immune thrombocytopenic purpura (ITP) $(n=1)$, diabetes mellitus, Guillain Barre syndrome $(n=1)$ and Crohn disease $(n=1)$ respectively.

Genetic disorders: Six (18\%) patients was found to have genetic disorders, trisomy $21(\mathrm{n}=2), 22 \mathrm{q} 11.2$ deletion $(n=1)$, 1p deletion $(n=1)$, Cohen syndrome $(n=1)$ and CHARGE syndrome $(n=1)$.

Among sIgMD patients who were younger than five years old, two more patients had the diagnosis of partial DiGeorge syndrom.

Atopy: Five patients $(15,1 \%)$ had asthma, and one was wheezy infant. One out of 5 patients was treated also for allergic rhinitis. 
Others: One patient was diagnosed with osteopetrosis, 12 patients $(36,3 \%)$ had sIgMD with no additional disorder or disease.

\section{Laboratory Findings}

Complete blood counts: Lymphopenia was detected in fifteen $(35,7 \%)$, neutropenia was detected in fourteen (33,3\%), leukopenia was seen in ten $(23 \%)$ and hypereosinophilia was seen in four patients $(9,5 \%)$.

Immunoglobulin levels: The IgG and IgA levels were in normal range as all the patients fullfilled the sIgMD criteria. Six (18\%) patients have high IgE levels. Immunoglobulin levels of patients are given in Supplementary Table 1.

Lymphocyte subsets: They were in normal range on admission in all patients.

\section{Antibody Response:}

Isohemaglutinin levels were normal (above 1/8) in 21 patients (63.6\%), and was not evaluated in others.

Anti-HBs antibody was positive in $24(72.7 \%)$ patients, and negative in $6(18.1 \%)$. Four patients were evaluated again after vaccination, two of the patients's antiHbs results were positive and two of them were still negative after vaccination. Two patients were not evaluated for antiHbs antibody after vaccination.

Treatment: None of the patients with sIgMD required intravenous immunoglobulin (IVIG). Nine patients $(27,2 \%)$ were given prophylactic antibacterial therapy for recurrent infections.

\section{Primary Immunodeficiency Diseases}

Although sIgMD is defined as isolated low levels of IgM with normal levels of IgA and IgG without other defined immunodeficiency, we also evaluated patients with welldefined PID. During follow-up, 13 patients were diagnosed with other types of PID diseases. These were T cell deficiency $(\mathrm{n}=6)$, combined immunodeficiency (CID) ( $\mathrm{n}=3$ ), autoimmune lymphoproliferative disease (ALPS) $(\mathrm{n}=2)$, chronic granulomatous disease $(\mathrm{n}=1)$, congenital neutropenia $(H A X-1$ gene defect $)(\mathrm{n}=1)$. The molecular defects of the patients diagnosed with CID in the follow-up were Serine-threonine kinase-4 $\left(S T K_{4}\right)$ deficiency $(\mathrm{n}=2)$, interleukin-2 inducible kinase $(I T K)$ deficiency $(\mathrm{n}=1)$. TwoSTK- 4 deficient patients were initially presented with susceptibility to infection and vasculitis; they got the molecular diagnosis of $\mathrm{T}$ cell deficiency approximately 2 years after admission. One patient developed acute EBV-associated lymphoproliferative syndrome. Two patients who were followed up with CID developed non-Hodgkin's lymphoma over years. Among the two patients died; one died at 22 years of age, had STK 4 gene mutation, lymphoma and amyloidosis; the other patient developed secondary malignancy (acute myeloid leukemia (AML) after treatment for lymphoma, and died at the age of 12 years.

\section{Discussion}

Selective IgM deficiency has been characterized as a rare form of PID, defined as a low serum IgM level (less than 2SDs below healthy controls), and normal serum IgA and IgG levels [14]. There are over 300 cases published in the literature. The prevalence ranges from $0.03 \%$ in a community-based study to $3 \%$ in allergy and immunology clinics $[15,8,9]$. In our study, the ratio of sIgMD is found as $0.12 \%$ among the patients that were followed up in pediatric immunology out-patient clinic for a definite period (33 out of 27,000 patients) [6].

SIgMD was first described by Hobbs et al in 1967 in a case report of two patients with fulminant meningococcal meningitis [7]. Although URTI were the most common clinical symptoms in sIgMD in literature, the patients presented also with various other infections; such as sepsis and meningitis [4]. In our series, URTI $(60.6 \%)$ were the most common type of infection, followed by otitis media $(42,4 \%)$ and pneumonia $(36,3 \%)$. 
It has been reported in the literature that sIgMD is more common in male sex. In our series, $74 \%$ of our patient population was male in accordance with the literature [6]. There are publications showing that sIgMD is more common in atopic and allergic people, and $\operatorname{IgE}$ levels may be high in patients with sIgMD [11]. In our study, six patients (13\%) had high IgE levels; five had atopy.

In Goldenstein's retrospective cohort, 36 adults with sIgMD were evaluated, the frequency of presentation symptoms were; recurrent URTI in $77 \%$; asthma in $47 \%$; allergic rhinitis in $36 \%$; vasomotor rhinitis in $19 \%$; angioedema in 14\%; and anaphylaxis in $11 \%$, and there was no patient who developed lymphoproliferative disease, or panhypogammaglobulinemia, and none died of life-threatening infections, malignancy, or fulminant autoimmune-mediated diseases [15]. Chovancova presented another adult cohort with 17 sIgMD patients. Most common manifestations were increased susceptibility to infections, especially involving recurrent URTI, pneumonia, urinary tract infections, sinusitis, otitis media and furuncles [10]. Allergic disorders included allergic rhinitis, drug allergy, bronchial asthma, atopic dermatitis, urticaria. Autoimmune manifestations including Sjögren's syndrome, systemic lupus erythematosus (SLE), thyreopathy and alopecia; malignancies including rectal adenocarcinoma, melanoma, and thymoma were also seen in this cohort [10]. Yel and her friends reported 15 adult symptomatic patients, their findings were similar with the previous studies, but they pointed out that impaired specific antibody response to pneumococcal antigens was seen in half of the patients, and IgM-deficient patients who present with recurrent/severe infections may benefit from immunoglobulin treatment, particularly in the presence of impaired pneumococcal antibody responses [16]. The results of our cohort was compatible with these reports. We observed infection susceptibility, chromosomal diseases, autoimmune/inflammatory, and allergic diseases associating with sIgMD. According to our findings, the follow-up of patients with low IgM is very important as about $\frac{1}{4}$ has got the diagnosis of PID in the follow-up. IgM deficiency may accompany CID [17]. The reason that we emphasized the group of 13 patients with defined PID was to highlight the low IgM levels that we come across in these group of patients.

Clinical associations of sIgMD with 22q11.2 deletion (DiGeorge Syndrome), ring chromosome 18 and chromosome 1 deletion were reported in literature [18]. The reason of the association of sIgMD and these deletions is not yet known, their association may also be a coincidence [11], or the genetic background of sIgMD may be related with these genetic locations. In our study; nine patients had an atypical facial appearance. Among five $(14 \%)$ patients with syndromes, two patients had trisomy 21 , one patient was diagnosed with DiGeorge syndrome, one had Cohen syndrome ( $\mathrm{COH}-1$ mutation), and one had $1 \mathrm{p}$ deletion.

SIgMD criteria of ESID includes normal IgG subclass levels, and normal vaccination responses with low IgM levels [5]. In some case series IgG subclass deficiency was detected in one fourth of patients [19, 15]. Hepatitis B vaccination response of six (18.1\%) patients were negative in our cohort. Janssen et al classifies selective IgM deficiency as follows: "true sIgMD" who met ESID registry criteria, "possible sIgMD" where ESID registry criteria were not fulfilled completely because data on IgG subclasses and/or vaccination response were not available, and "unclassified primary antibody deficiency" since other abnormalities of antibodies, IgG subclass deficiency, and/or impaired response to vaccine were present [20]. Patients of our cohort meet "possible sIgMD" according to this classification due to lack of Ig G subclass and vaccination responses. Gupta et al. explained that terminology of "true", "possible", and "unclassified" selective IgM deficiency should not be used, because this definition would exclude a sizeable number of patients, including those with complete absence of serum IgM, from the diagnosis of selective IgM deficiency [21]. Thus, we think that selective IgM deficiency should be defined only according to serum antibody levels and age [21].

In addition to providing early defense against microbes, IgM plays an important role in immune homeostasis, and provides protection from consequences of autoimmunity and inflammation [9]. There is an increased prevalence of autoimmune diseases in patients with sIgMD [7]. Autoimmune diseases associated with sIgMD are SLE, rheumatoid arthritis, Hashimoto's thyroiditis, autoimmune hemolytic anemia (AHA), Celiac disease, vitiligo, polymyositis, autoimmune glomerulonephritis, and chronic ITP [7]. In our cohort, six patients $(9,5 \%)$ with sIgMD was also diagnosed with autoimmune and inflammatory disease, such as, Behcet's disease, ITP, Gullian-Barre syndrome, diabetes mellitus and Crohn disease. According to the reports, autoimmune diseases 
are more common in adults rather than children [9]. In our cohort, two out of four patients with autoimmune diseases were older than eighteen years old; one of them had Behçet's diseases, the other had ITP.

A number of malignancies were described in SIgMD patients as case series, including clear cell sarcoma, nonHodgkin's lymphoma, promyelocytic leukemia and hepatocellular carcinoma [7]. In our series, malignancy developed in three $(8,8 \%)$ patients, they were AML, neuroblastoma and tubuler adenoma in sigmoid colon.

There are also reports in the literature showing the relationship between sIgMD and chronic inflammatory diseases, such as Crohn, ulcerative colitis and Celiac disease associating protein-losing enteropathy. We don't know the reason why only IgM levels, not IgG levels, decreased in patients with protein loosing enteropathy. All these diseases may possibly associate with PID. Some sIgMD patients, having IgM levels improved after treatment of primary disease (e.g. gluten-poor diet) have also been reported. In our cohort, there was one patient with Crohn disease, but there was no improvement in the IgM levels after the treatment [7].

Intravenous immunoglobulin treatment's beneficial effects on sIgMD patients were reported [16]. Goldenstein et al. showed clinical improvement with high dose IVIG in patients with comorbidity of asthma and bronchiectasis. In our cohort, none of the patients with sIgMD needed IVIG treatment.

Immunologic evaluation should be recommended to the family members who had frequent infections and autoimmune, inflammatory and lymphoproliferative/malign disease.

As a result, the etiology, pathogenesis, clinical course of sIgMD is not clear yet. In the literature, only about three percent of patients with sIgMD were completely asymptomatic both in the adult age and in pediatric age groups; whereas in our series this rate was $24 \%(n=8)[15,11]$. We observed in our series that about $\frac{1}{4}$ had PID, and $10 \%$ had CID. These findings should be supported by further studies. Patients should have regular follow-up in immunology clinics even if they do not have clinical symptoms.

\section{References}

1. Goldstein MF, Goldstein AL, Dunsky EH, Dvorin DJ, Belecanech GA, Shamir K. Pediatric selective IgM immunodeficiency. Clin Dev Immunol. 2008;2008:624850. doi:10.1155/2008/624850.

2. Geier CB, Sauerwein KMT, Leiss-Piller A, Zmek I, Fischer MB, Eibl MM et al. Hypomorphic Mutations in the BCR Signalosome Lead to Selective Immunoglobulin M Deficiency and Impaired B-cell Homeostasis. Front Immunol. 2018;9:2984. doi:10.3389/fimmu.2018.02984.

3. Guill MF, Brown DA, Ochs HD, Pyun KH, Moffitt JE. IgM deficiency: clinical spectrum and immunologic assessment. Ann Allergy. 1989;62(6):547-52.

4. Hobbs JR, Milner RD, Watt PJ. Gamma-M deficiency predisposing to meningococcal septicaemia. $\mathrm{Br}$ Med J. 1967;4(5579):583-6. doi:10.1136/bmj.4.5579.583.

5. Seidel MG, Kindle G, Gathmann B, Quinti I, Buckland M, van Montfrans J et al. The European Society for Immunodeficiencies (ESID) Registry Working Definitions for the Clinical Diagnosis of Inborn Errors of Immunity. J Allergy Clin Immunol Pract. 2019;7(6):1763-70. doi:10.1016/j.jaip.2019.02.004.

6. Cassidy JT, Nordby GL. Human serum immunoglobulin concentrations: prevalence of immunoglobulin deficiencies. J Allergy Clin Immunol. 1975;55(1):35-48. doi:10.1016/s0091-6749(75)80006-6.

7. Louis AG, Gupta S. Primary selective IgM deficiency: an ignored immunodeficiency. Clin Rev Allergy Immunol. 2014;46(2):104-11. doi:10.1007/s12016-013-8375-x.

8. Zhang Y, Garcia-Ibanez L, Toellner KM. Regulation of germinal center B-cell differentiation. Immunol Rev. 2016;270(1):8-19. doi:10.1111/imr.12396.

9. Gupta S, Gupta A. Selective IgM Deficiency-An Underestimated Primary Immunodeficiency. Front Immunol. 2017;8:1056. doi:10.3389/fimmu.2017.01056. 
10. Chovancova Z, Kralickova P, Pejchalova A, Bloomfield M, Nechvatalova J, Vlkova M et al. Selective IgM Deficiency: Clinical and Laboratory Features of 17 Patients and a Review of the Literature. J Clin Immunol. 2017;37(6):559-74. doi:10.1007/s10875-017-0420-8.

11. Ross IN, Thompson RA. Severe selective IgM deficiency. J Clin Pathol. 1976;29(9):773-7. doi:10.1136/jcp.29.9.773.

12. Justiz Vaillant AA, Wilson AM. Transient Hypogammaglobulinemia of Infancy. StatPearls. Treasure Island (FL): StatPearls Publishing

Copyright (C) 2020, StatPearls Publishing LLC.; 2020.

13. Ameratunga R, Ahn Y, Steele R, Woon ST. Transient hypogammaglobulinaemia of infancy: many patients recover in adolescence and adulthood. Clinical and experimental immunology. 2019;198(2):224-32. doi:10.1111/cei.13345.

14. Haddad ZH, Allen RF, Towner JW, Wilson MG. IgA, IgM, and partial deletion of chromosome 18. Lancet. 1969;1(7596):678. doi:10.1016/s0140-6736(69)92044-3.

15. Goldstein MF, Goldstein AL, Dunsky EH, Dvorin DJ, Belecanech GA, Shamir K. Selective IgM immunodeficiency: retrospective analysis of 36 adult patients with review of the literature. Ann Allergy Asthma Immunol. 2006;97(6):717-30. doi:10.1016/s1081-1206(10)60962-3.

16. Yel L, Ramanuja S, Gupta S. Clinical and immunological features in IgM deficiency. Int Arch Allergy Immunol. 2009;150(3):291-8. doi:10.1159/000222682.

17. Soulas-Sprauel P, Rivera-Munoz P, Malivert L, Le Guyader G, Abramowski V, Revy P et al. V(D)J and immunoglobulin class switch recombinations: a paradigm to study the regulation of DNA end-joining. Oncogene. 2007;26(56):7780-91. doi:10.1038/sj.onc.1210875.

18. Al-Herz W, McGeady SJ, Gripp KW. 22q11.2 deletion syndrome and selective IgM deficiency: an association of a common chromosomal abnormality with a rare immunodeficiency. Am J Med Genet A. 2004;127a(1):99-100. doi:10.1002/ajmg.a.20639.

19. Lucuab-Fegurgur DL, Gupta S. Comprehensive clinical and immunological features of 62 adult patients with selective primary IgM deficiency. American journal of clinical and experimental immunology. 2019;8(6):55-67.

20. Bousfiha A, Jeddane L, Picard C, Ailal F, Bobby Gaspar H, Al-Herz W et al. The 2017 IUIS Phenotypic Classification for Primary Immunodeficiencies. J Clin Immunol. 2018;38(1):129-43. doi:10.1007/s10875-0170465-8.

21. Gupta S, Gupta A. Defining Primary Selective IgM Deficiency. J Clin Immunol. 2019;39(4):350-2. doi:10.1007/s10875-019-00641-4.

\section{Hosted file}

figür.pdf available at https://authorea.com/users/368348/articles/487512-selective-igmdeficiency-follow-up-and-outcome

\section{Hosted file}

tablo.pdf available at https://authorea.com/users/368348/articles/487512-selective-igmdeficiency-follow-up-and-outcome 